\title{
Spectral Imaging Technique for Visualizing the Invisible Information
}

\author{
Shigeki Nakauchi \\ Department of Information \& Computer Sciences, \\ Toyohashi University of Technology, \\ Toyohashi 441-8580, Japan \\ naka@bpel.ics.tut.ac.jp \\ http://www.bpel.ics.tut.ac.jp
}

\begin{abstract}
Importance of multi-spectral colour information has been remarkably increasing in imaging science. This is because the original spectrum contains much more information about the surface of target objects than perceived colour by human. This article describes our attempts to visualize the invisible information, such as the constituent distribution and internal microstructure of food and plant responses to the environmental stress, by a spectral imaging technique.
\end{abstract}

\section{Introduction}

More than 300 years ago, Sir Isaac Newton first discovered the spectrum. In his famous experiments, he separated daylight into its spectral components by passing it through a prism. Although we can see several different colours in the spectrum, Newton also claimed that "light itself is not coloured", meaning that colour results from our visual function to encode the spectral information. Our mechanism to see colours originates in capturing the incident light by three types of colour sensors in the retina which can signal the spectral information by its relative activity. Due to this trichromatic nature, human can see only a portion of the spectral information. Even if the two incoming lights have different physical characteristics, we can not distinguish them when outputs of the three types of photo sensors are the same.

The limitation of our ability to capture the spectral information is owing to the number and the wavelength range of color sensors. Certain kinds of animals can see more colors than human because of more color sensors, e.g. five different colour sensors of Butterflies or a UV sensor of bees [1], [2]. In this sense, if we could develop an artificial eye with higher wavelength resolution and wider wavelength range, it can be superior to our visual system.

Spectral imaging is expected to be a key technology for such an artificial hypervision system. This article demonstrates our several attempts to visualize the information which is invisible to human using the spectral imaging technique, such as the constituent distribution and internal microstructure of food, and plant responses to the environmental stress caused by ozone. 


\section{Visualization of the Sugar Content Distribution of Melons by Near-Infrared Imaging Spectroscopy}

\subsection{Background}

Recently, automated sweetness sorting machines for vegetables or fruits are getting common, and now in use in more than 1000 packing houses in Japan. However, because of uneven distribution of sugar content, some fruits sorted by this type of machine as sweet ones taste insipid. This is a limitation in point-measurement, and instead imaging-based measurement is required. We aim to develop a technique for visualization of the sugar content distribution of a melon by NIR imaging.

The spectral imaging system we are using consists of an acousto-optic tunable filter (AOTF) and a peltier-cooled monochromatic CCD camera as shown in Fig.1. It can measure the spectral information at any pixel position, ranging from $400 \mathrm{~nm}$ to $1,000 \mathrm{~nm}$ at about $2 \mathrm{~nm}$ intervals, instead of three channel values (, $\mathrm{G}$ and $\mathrm{B}$ ) as in conventional digital cameras.

Fig. 1. Spectral imaging system consisting of an acousto-optic tunable filter (AOTF, a solid-state electronically tunable spectral bandpass filter) and a peltier-cooled monochromatic CCD camera. This system can measure spectral images ranging from $400 \mathrm{~nm}$ to $1,000 \mathrm{~nm}$ at about $2 \mathrm{~nm}$ intervals

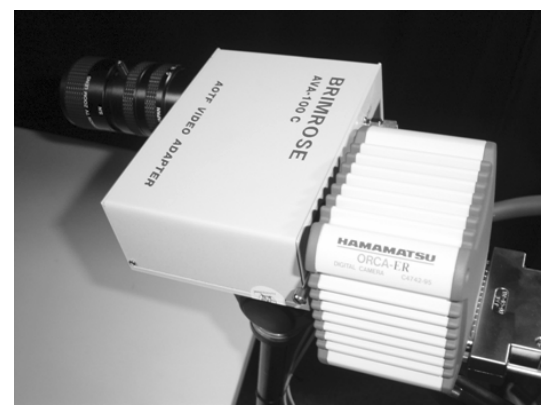

\subsection{Method and Results}

In order to predict the sugar content from measured spectra, we need to make a calibration data set, which is a pair set of absorption spectra and sugar content data (Brix) measured by a refractometer. To do this, we cut and pick up a set of sample slice from melons, measure the absorption spectra and the Brix values of slices as shown in Fig.2. By repeating this process, we obtain a calibration data set shown in Fig.3. Then we describe the relation between the absorbance and the Brix by a multivariate regression analysis. For the data set obtained from 835 samples, we could get a relatively high precision $(\mathrm{r}=0.89)$.

Once we have a calibration curve, the measured absorption spectra at each pixel can be mapped into the sugar content value. Fig.4 shows an example of sugar content map. From the map, we can see the difference of sugar content distribution among two varieties of melons. For example, the green and red type of varieties had different distribution, that is, the red one has more uniform sugar content distribution than the green one. 


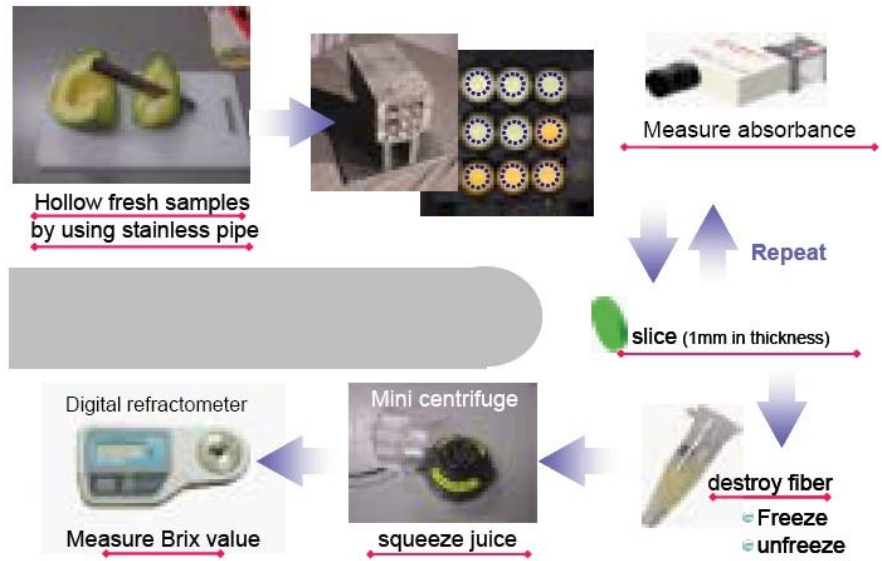

Fig. 2. Process for making calibration data to estimate the Brix value from spectral absorbance measured by the spectral imaging system
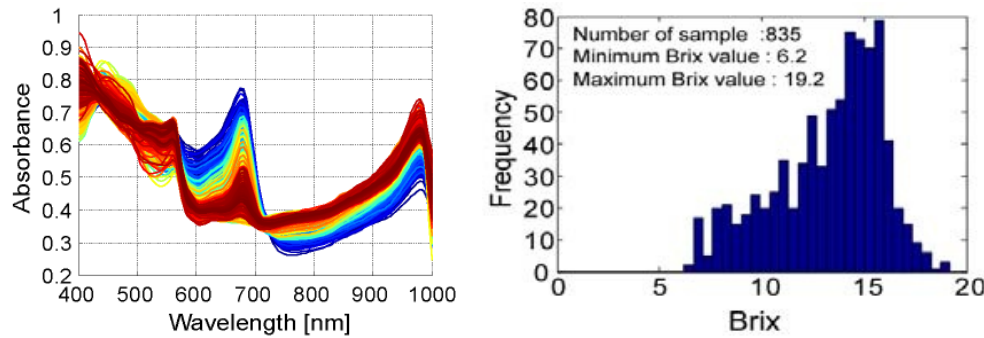

Fig. 3. Absorbance spectra (left) and Brix values (right shown as histogram) obtained from 835 samples. Colour of a curve represents the Brix values as red for high and blue for low Brix values. A multivariate regression analysis was applied to pairs of these data sets to construct a calibration curve for the sugar content
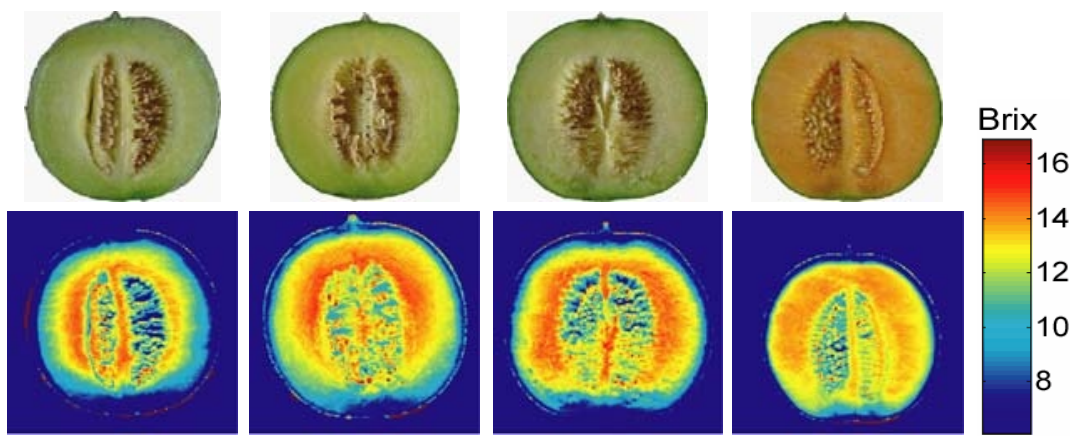

Fig. 4. Visualization of sugar content distributions for cut in half melons using spectral imaging technique. From the sugar content maps shown as bottom panels, the green and red types of varieties had different distributions 


\section{Visualization of the Internal Microstructure of Soybeans by Spectral Fluorescence Imaging}

\subsection{Background}

Soybeans (Glycine soja L.) are a very fast growing sector of agricultural production in the world market including western world, and many researches have been done on measurement of constituents to understand and improve the soybeans' quality. Here we focus on the excitation-emission matrix (EEM) to visualize the internal microstructure of soybeans.

\subsection{Method and Results}

An EEM is a contour-like graph composed of an excitation wavelength axis, an emission wavelength axis and a fluorescence intensity axis. Every constituent can be specified by EEM pattern matching because it has a unique EEM pattern reflecting its characteristics. EEM measurements and analyses have been carried out in the archaeological field or the environmental assessment to specify old dyes on the paintings or pollutant in the sea water [3], [4]. Although there have been only a few studies of EEM application to food, it is expected that the internal structure and constituent distribution, which greatly affect food qualities, can be visualized by the measurement and analysis of an EEM at any point of food.

Measurement system composed of a micro-slicer, a spectral illuminator and a spectral imaging system as shown in Fig.5. The spectral illuminator consists of a xenon lamp and a grating spectrometer. It illuminates the sample surface with light at any wavelength from $200 \mathrm{~nm}$ to $1,000 \mathrm{~nm}$. The spectral imaging system is made up with a liquid crystal tunable filter and a monochromatic CCD camera. The sample surface image can be captured at any wavelength from $400 \mathrm{~nm}$ to $1,100 \mathrm{~nm}$. An EEM at any point of the sample can be measured by combining the spectral illuminator and spectral imaging system.

Process for visualizing the internal microstructure of soybeans is shown in Fig.6. The sample soybean was cut in half by the micro-slicer and fluorescence images of the cut surface were captured for various combinations of excitation and emission wavelengths. The excitation wavelength was changed from $350 \mathrm{~nm}$ to $570 \mathrm{~nm}$ at $10 \mathrm{~nm}$ intervals and the emission wavelength was from $400 \mathrm{~nm}$ to $600 \mathrm{~nm}$ at $10 \mathrm{~nm}$ intervals. 273 images were captured in total as shown in Fig.6(a).

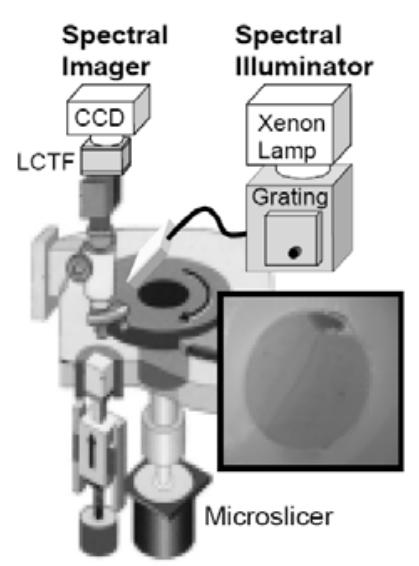

Fig. 5. Measurement set up for EEM imaging, consisting of a micro-slicer, a spectral illuminator and a spectral imaging system 
The EEM at each pixel of the cut in half soybean image was calculated from measured fluorescence images as shown in Fig.6(b). In order to quantitatively clarify the difference among EEM patterns of different pixels, Principle Component Analysis (PCA) was applied to the EEM of each pixel. PCA is a statistical method to compress multidimensional data into two- or three-dimensional coordinate with the data variance maximized. Using this method, each pixel was plotted on a PC score plot according to its EEM pattern as shown in Fig.6(c).

As shown in Fig.6(d), the PC score plot was converted into a CIELAB color space in order to assign to each pixel. That is, if two pixels had similar EEM patterns, similar colors were assigned to these pixels and if not, they were assigned different colors in proportion to the EEM pattern difference. By applying this color mapping to each pixel, a color distribution map was developed as shown in Fig.6(e).

It is clearly seen in Fig.6(d) that the aleurone layer, the vein-like structure and the embryo were assigned as blue, green and red respectively so that they could be clearly distinguished in the color distribution map as shown in Fig.6(e). Especially, a characteristic branching of the vein-like structure could be clearly observed showing that the measurement and visualization method based on EEM is useful for the visualizing the internal structure in food.

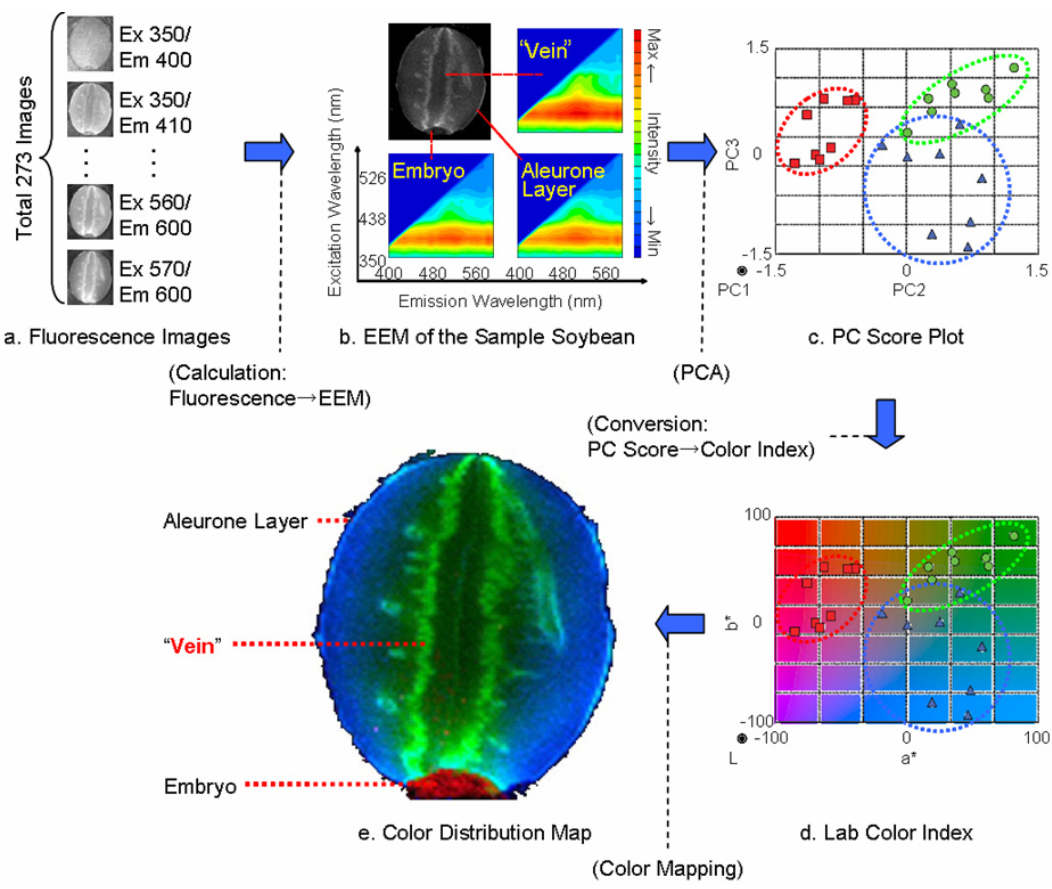

Fig. 6. Visualization of the internal microstructure of soybeans by EEM imaging 


\section{Visualization of Early Ozone Stress Response in Leaves}

\subsection{Background}

Ozone on the surface of the earth, that is toxic to human beings, animals and plants, is increasing worldwide [5]. Even in case when concentration of ozone is relatively low for human beings, plants receive so-called ozone stress resulting in such as low growth [6] and visible injury [7]. Especially visible foliar injury is a matter of great importance to farmers because agricultural products with visible foliar injury are not marketable and it causes serious economic loss [8]. Generally, this type of injury cannot be mitigated by common measures of plant protection. There is a possibility, however, of reducing the visible injury by fertilizer management [8] or avoiding additional watering of the plants [9]. During shortage of water, plants tend to keep closed their stomata in the leaf surface that allows ozone to enter into the leaf. Less ozone uptake means less ozone damage. Therefore, it is an important subject to detect the influences of ozone stress in its early stages and avoid visible injury by well-timed cultivation measures.

Plant physiological status has been investigated using spectral reflectance at landscape to a single leaf level [10], [11]. With the advantage of technology, more precise investigation of plant physiological status using spectral imaging devices is nowadays possible [12]. To this end, we present a method for detecting and visualizing the plants stress before the occurring of the visible foliar injury using spectral imaging technique.

\subsection{Materials and Methods}

Radish (Raphanus sativus L. cv. Akamaru) which is relatively sensitive to ozone [13], [14] was used as a test material in the experiment. The seed was planted in 900 $\mathrm{ml}$ flowerpots and was cultivated under ozone-free environment for 38 days. Two potted plants were selected for the ozone exposure and control experiment. Ozone exposure was done in a laboratory environment and sets of spectral images of the leaves were measured. The potted plant was exposed to ozone twice (time 0-16 and 49-64, respectively) and was set in the ambient air after the exposure. Spectral images were measured at every 4 or 5 hours during the exposure and at every 10 hours after the exposure.

Ozone was produced with an ultraviolet (UV) lamp in an ozone generator, and the gas containing ozone was purified by water filter and was led into a glass chamber in which the potted plant was set. The concentration of ozone was controlled by adjusting the voltage applied to the UV lamp. Fluorescent lights $(7200 \mathrm{~K})$ were used for illuminating the potted plants so that ozone sensitivity in plants became high. The reason for this is that plants tend to keep opened their stomata in the leaf surface that allow ozone to enter into the leaf when the intensity of illumination is high. The potted plant for control experiment was set just beside the glass chamber during ozone exposure and was set in the ambient air after exposure time.

Then the potted plant was taken out of the glass chamber and the leaves were set on an experimental table covered with black cloth. An intensity image of the leaves 
illuminated by halogen lamps was taken with a monochrome CCD camera through AOTF with transmitting wavelengths ranging from $400 \mathrm{~nm}$ to $1,000 \mathrm{~nm}$. To calculate spectral reflectance images, a spectral image of a white board (BaSO4) was used as the reference white.

It is well-known that spectral reflectance of green leaves shows typical shape, such as absorption in a long-wavelength region and plateau in near infrared region. We here focus on the averaged reflectance values in the visible and invisible wavelength bands defined as RED (670-690 $\mathrm{nm})$ and NIR $(780-800 \mathrm{~nm})$, in which plant physiological status is expected to be reflected, and the relative changes of those values $(\triangle R E D$ and $\triangle N I R$ ) from the initial status due to the ozone exposure were analyzed.

\subsection{Results}

Fig.7 shows photos of leaves (A) exposed to ozone and (B) without exposure as control data. These photos were taken with a digital camera at time 0 (beginning of the ozone exposure), 16, 54, 59 and 131 hours. The leaves exposed to ozone were gradually changed during the first ozone exposure (time 0-16), however no visible injury occurred. At time 59 during the second ozone exposure (time 49-64), visible injury occurred (shown by a left arrow in Fig.7 (A)) while the controlled leaves did not show any remarkable changes. The visible injury called as necrosis is one of the common symptoms due to ozone exposure. In this case, the colour between veins of green leaves changed to white due to the collapse of leaf tissue. Visible injury developed after the second ozone exposure was terminated, and white spots were clearly found at time 131 (shown by a right arrow in Fig.7 (A)).

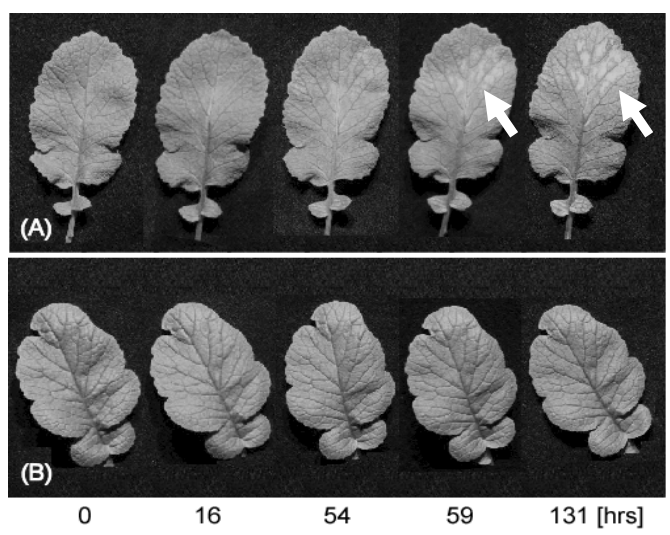

Fig. 7. Radish leaves (A) exposed to ozone and (B) without exposure (control). Visible injury first occurred at time 59, while the control leaves did not show any remarkable changes

To analyze the influences of the ozone exposure to leaves, spectral images were measured for the severely visible injured area of the leaf and these were compared 
with that of the controlled leaf as follows. First, severely visible injured area was manually decided by referring to the photo taken at time 131 . Then, RED and NIR data at time 0 to 131 were extracted from the decided injured area. The RED and NIR data were averaged in the area, and $\triangle R E D$ and $\triangle N I R$ were calculated so that the initial $R E D$ and NIR data were brought to zero by subtracting them from data.

Fig.8 (a) and (b) show the relative changes of RED and NIR of the leaves exposed to ozone and controlled leaves, $\triangle R E D$ and $\triangle N I R$, respectively. It was found that (1) $\triangle N I R$ increased more than $\triangle R E D$ during the first ozone exposure. (2) $\triangle N I R$ approached to zero when the exposure to ozone was terminated before visible injury occurred at time 59. It was also found that $\triangle R E D$ increased remarkably and visible injury occurred during the second ozone exposure. After visible injury occurred, $\triangle R E D$ continued to increase and $\triangle N I R$ decreased. Controlled leaves did not show any regular changes. Thus, $\triangle R E D$ is clearly consistent with our observations, while $\triangle N I R$ may reflect invisible changes of leaves due to the ozone exposure.

To investigate how $\triangle R E D$ and $\triangle N I R$ actually relates to the visible injury of the leaves, distributions of these values in whole leaf are visualized. Fig.9 shows the visualization results. Note that, in these images, average of $R E D$ and NIR values at time 0 were subtracted from each image to show the relative changes from the initial condition. In $\triangle R E D$ images, visible foliar injury occurred at time 59 which is consistent with our observations. While, in $\triangle N I R$ images, remarkable changes are already found at time 4 , at almost the same area where visible injury occurred. The controlled leaves did not show any remarkable changes. We can conclude that (3) $\Delta N I R$ showed remarkable change in parts where visible injury occurred earlier than $\triangle R E D$. Therefore, the proposed method can be used for detecting the ozone stress in its early stages which allow us to protect the plants from the ozone stress and avoid the visible injury.
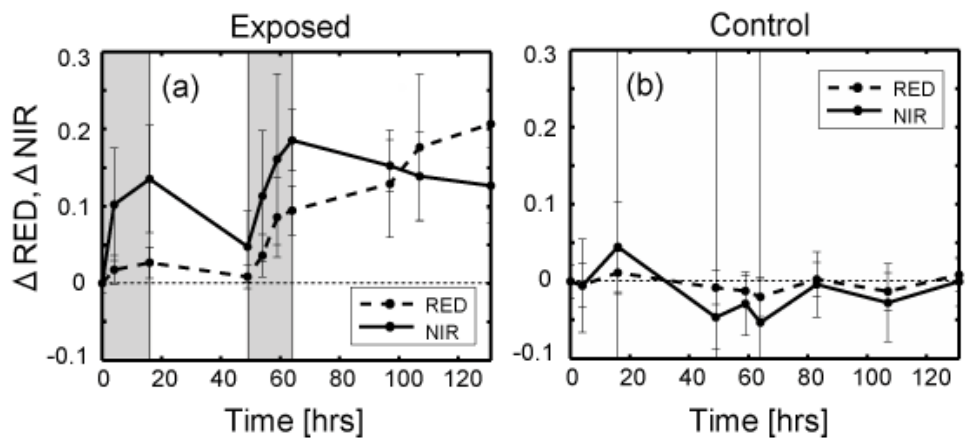

Fig. 8. Dynamic changes of $\triangle R E D$ and $\triangle N I R$. (a) Exposed to ozone and (b) controlled. During the first exposure (time 0-16), $\triangle N I R$ increased more than $\triangle R E D$. When the exposure was terminated (time 16-49), $\triangle N I R$ approached zero. During the second exposure (time 49-64), $\triangle R E D$ increased remarkably and visible injury occurred at time 59. Control did not show any regular changes 


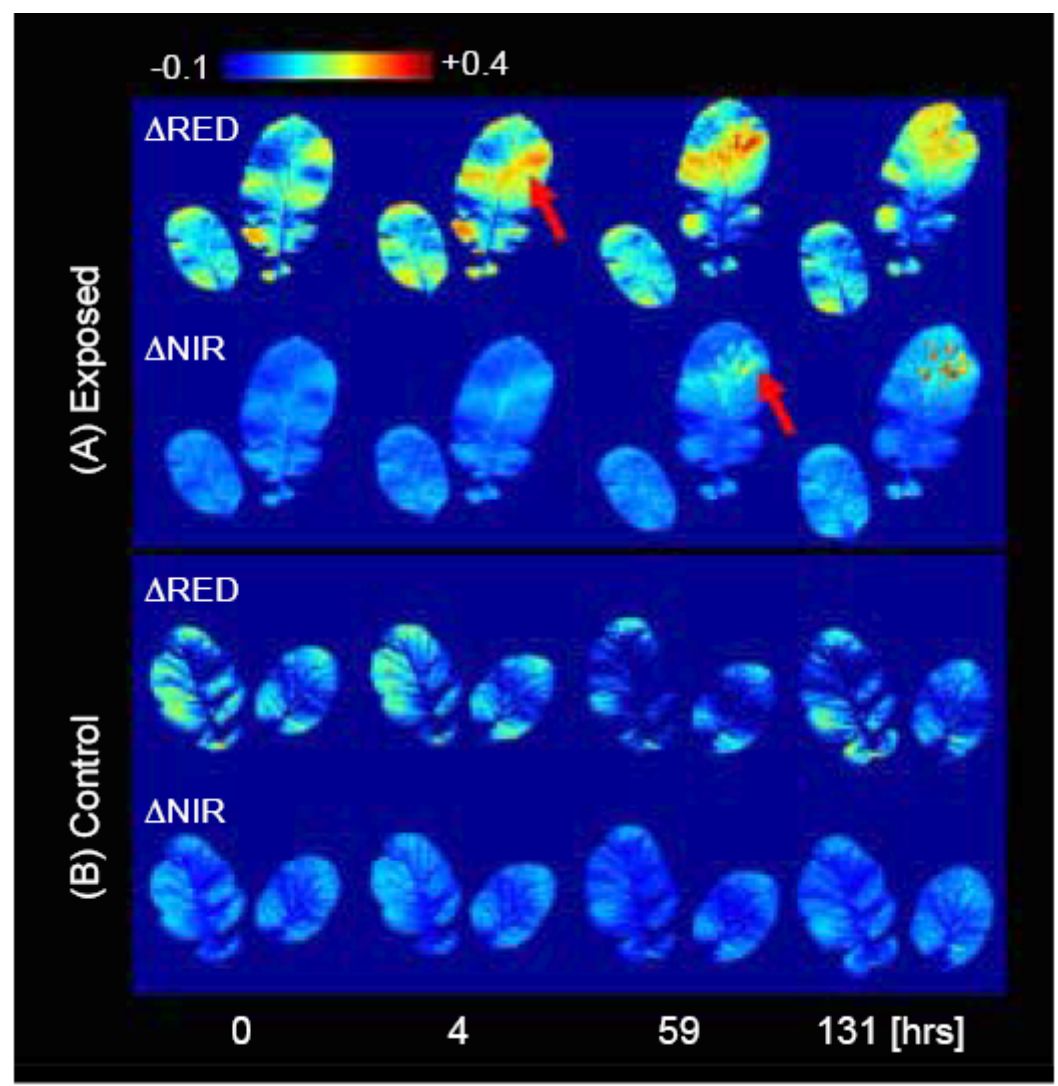

Fig. 9. Changes of reflectance in visible $(\triangle \mathrm{RED})$ and invisible $(\triangle \mathrm{NIR})$ wavelength bands of leaves (A) exposed to ozone and (B) without exposure. At time 59, $\triangle \mathrm{RED}$ changed the area where visible injury occurred. At time 4 , however, $\triangle$ NIR already showed remarkable change at almost the same area. The controlled leaves did not show any remarkable changes

\section{Conclusions}

In this article, several applications of spectral imaging technique were demonstrated, mainly focusing on the visualization of the invisible information of food and plants. Spectral imaging technique is characterized by its ability to capture enormous amount of information. Due to recent progress of the optical technology, several equipments for the spectral imaging allow us to do measurements in a laboratory scale. It is expected that the spectral imaging technique can be applied to several different areas. At the same time, importance of processing / analyzing / storing / transmittance of the measured spectral images may also increase. 


\section{Acknowledgements}

Author thanks T.Suzuki for his efforts on measurements of spectral images of melons; Dr. Sugiyama and Dr. Tsuta for EEM measurements of soybeans; Dr. Miyazawa and H.Iwasaki for measurements of plant responses to ozone. This work was partially supported by Cooperation of Innovative Technology and Advanced Research in Evolutional Area, and the 21st Century COE Program "Intelligent Human Sensing".

\section{References}

1. Kinoshita, M., Shimada, N., Arikawa, K.: Colour vision of the foraging swallowtail butterfly papilo xuthus. J.Exp.Biol. 202 (1999) 95-102

2. 2 Menzel, R., Shmida, A.: The ecology of flower colurs and the natural colour vision of insect pollinators: The Israeli flora as a study case. Biol. Rev. 68 (1993) 81-120

3. Shimoyama S., Noda Y., Katsuhara S.: Non-destructive Determination of Colorants Used for Traditional Japanese Ukiyo-e Woodblock Prints by the Three-dimensional Fluorescence Spectrum Using Fibre Optics. Bunseki Kagaku 47(2) (1998) 93-100

4. Booksh K. S., Muroski A. R., Myrick M. L.: Single-Measurement Excitation/ Emission Matrix Spectrofluorometer for Determination of Hydrocarbons in Ocean Water. 2. Calibration and Quantitation of Naphthalene and Styrene. Analytical Chemistry 68(20) (1996) 3539-3544

5. Akimoto, H.: Global air quality and pollution, Science 302 (2003) 1716-1719

6. Gregg, J.W., Jones, C.G., Dawson, T.E., Urbanization effects on tree growth in the vicinity of New York City. Nature 424 (2003) 183-187

7. Novak, K., Skelly, J.M., Schaub, M., Kräuchi, N., Hug, C., Landolt, W., Bleuler, P.: Ozone air pollution and foliar injury development on native plants of Switzerland. Environ Pollut. 125(1) (2003) 41-52

8. Nouchi, I.: Agricultural countermeasures for avoiding crop injury from ozone in Japan. J. Agric. Meteorol. 59 (2003) 59-67

9. Kostka-Rick, R.: Biomonitoring, http://www.biomonitoring.com/e/ozon.html

10. Bowman, W.D.,: The relationship between leaf water status, gas exchange, and spectral reflectance in cotton leaves. Remote Sens. Environ 30 (1989) 249-255

11. Peñuelas, J. and Filella, I.: Visible and near-infrared reflectance techniques for diagnosing plant physiological status. Trends in Plant Science 3 (1998) 151-156

12. Aario, S., Kauppinen, H., Silvén, O. and Viilo, K.: "Imaging spectrography of greenhouse plants for vitality estimation," in Proceedings of the 3rd International Conference on Computer Vision Systems (ICVS 03), International Workshop on Spectral Imaging, 2002, pp. 49-55.

13. Kostka-Rick, R., Manning, W.J., Radish (Raphanus sativus L.): A model for studying plant responses to air pollutants and other environmental stresses. Environ Pollut. 82(2) (1993) 107-38

14. Izuta, T., Miyake, H., Totsuka, T.: Evaluation of air-polluted environment based on the growth of radish plants cultivated in small-sized open-top chambers. Environ Sci. 2 (1993) 25-37 